Modeling and simulation of DNA flow in a microfluidic-based pathogen detection system

D. Trebotich, G. H. Miller

February 1, 2005

3rd Annual International IEEE EMBS Special Topic Conference on Microtechnologies in Biomedicine Oahu, HI, United States May 12, 2005 through May 15, 2005 
This document was prepared as an account of work sponsored by an agency of the United States Government. Neither the United States Government nor the University of California nor any of their employees, makes any warranty, express or implied, or assumes any legal liability or responsibility for the accuracy, completeness, or usefulness of any information, apparatus, product, or process disclosed, or represents that its use would not infringe privately owned rights. Reference herein to any specific commercial product, process, or service by trade name, trademark, manufacturer, or otherwise, does not necessarily constitute or imply its endorsement, recommendation, or favoring by the United States Government or the University of California. The views and opinions of authors expressed herein do not necessarily state or reflect those of the United States Government or the University of California, and shall not be used for advertising or product endorsement purposes. 


\title{
Modeling and simulation of DNA flow in a microfluidic-based pathogen detection system
}

\author{
D. Trebotich $\dagger$, G. H. Miller $\ddagger$ \\ $\dagger$ Center for Applied Scientific Computing, Lawrence Livermore National Laboratory, P.O. Box 808, L-560, Livermore, CA \\ 94551, USA \\ $\ddagger$ Department of Applied Science, University of California, One Shields Avenue, Davis, CA 95616, USA.
}

\begin{abstract}
We present simulation results from a new computational model of DNA flow in microfluidic devices. This work is important because computational models are needed to design miniaturized biomedical devices that are becoming the state-of-the-art in many significant applications including pathogen detection as well as continuous monitoring and drug delivery. Currently advanced algorithms in design tools are non-existent but necessary to understand the complex fluid and polymer dynamics involved in biological flow at small scales. Our model is based on a fully coupled fluid-particle numerical algorithm with both stochastic and deterministic components in a bead-rod polymer representation. We have applied this work to DNA extraction configurations in a microfluidic PCR chamber used in a pathogen detection system. We demonstrate our method on the test problem of flow of a single DNA molecule in a 2D packed array microchannel. We are also investigating mechanisms for molecular "sticking" using short range forces.
\end{abstract}

Keywords-DNA, polymer flow, pathogen detection, bioMEMS, PCR, microfluidics, modeling

\section{INTRODUCTION}

Modeling complex biological fuids is a challenge because these types of fbws are not well understood, and the constitutive behavior of these types of fluids is not easily represented. Modeling is further complicated when fbw is restricted to the microscale due to the presence of large particles in the fuid whose molecular lengths are comparable to the fbw geometry. For example, a highly concentrated solution of suspended polymer molecules may be represented at large scales with a continuum Oldroyd-B constitutive model (e.g., [1]). However, when the geometry length scales are comparable to the inter-polymer spacing a continuum approximation is no longer appropriate. Additionally, when the length scale of the geometry is comparable to the length of an individual polymer macromolecule,

This work was performed under the auspices of the U.S. Department of Energy by the University of California, Lawrence Livermore National Laboratory under contract No. W-7405-Eng-48. new physical behavior may be observed. Here, we are concerned with this dilute microscale limit, which finds application in sample processing and sensor technology. Our model will consider discrete polymers suspended in an incompressible viscous solvent.

We apply our model to DNA extraction in a polymerase chain reaction (PCR) chamber as part of a pathogen detection system. The primary extraction technique currently being pursued by our collaborators at LLNL is a packed bed reactor, which is essentially a small tube packed with microscale glass beads. The physical model presents a three-dimensional (3D) multiscale problem where DNA molecules must be resolved along with the fbw geometry. A second extraction design is a pillar chip which is an array of cylindrical obstructions in a shallow microchannel. Though a 3D problem this latter design lends itself to $2 \mathrm{D}$ models. The restriction to $2 \mathrm{D}$ is useful and can be reduced to model a single molecule traveling through a smaller section of the array. This is the model problem where we have honed the algorithm development to model relevant physics of the fbw for the full 3D problem.

The model problem offers possibilities for processing DNA-laden fluids. For example, when DNA-laden fluids fbw through an array of pillars, size separation may be achieved: longer molecules are slowed by their interaction with the pillars to a greater degree than are smaller molecules. Pillar rods may also be electro-chemically treated to promote selective binding of macromolecules, yielding a mechanism for sticking, which is a prerequisite for DNA amplification. Underlying fbw fields in the vicinity of complex geometry variations, as in a pillar array or abrupt contraction, display steep velocity gradients. The shear forces due to these gradients can be strong enough to break molecular bonds, a dynamic which can be useful in a PCR device but detrimental in a drug delivery system. The success of modeling such strategies relies critically on the fluid-molecule coupling. 


\section{METHOD}

Our approach is based on the fluid-particle coupling algorithm of Trebotich and Miller [2] for incompressible polymer-laden fbws in irregular microscale geometries. Polymers are represented as beads and rods, that is, a collection of point masses connected by constrained interparticle spacing. Each particle is subject to a hydrodynamic drag force from the fluid, and Brownian motion. The fluid in turn feels the effect of the particles via a cloud-in-cell model, which is a way to discretize the Dirac delta function. We note that the backward coupling is hardly felt due to the atomic nature of the particles (small mass), unless the Newtonian solution is concentrated with polymers. Irregular geometry boundaries are treated with an embedded boundary, or cutcell, Cartesian grid method [3], [4]. Incompressibility is enforced and velocity and pressure evolved by a projection method [5].

\section{RESULTS AND DISCUSSION}

The physical problem which we have set out to model is fbw of a DNA molecule in a packed bed reactor used for extraction in an amplification device. The packed bed is a small tube (approximate diameter $1 \mathrm{~mm}$ ) packed with glass microbeads (approximate diameter 50-100 $\mu \mathrm{m}$ ). This is a true $3 \mathrm{D}$ problem, and one for which we have obtained preliminary results of the continuum fbw without particle coupling. In the left image of Fig. 1 we show pressure data for continuum fbw in a cylinder with fixed beads obstructing the fbw. High-resolution simulation of this 3D problem will require additional scalability by making use of high performance computing such as distributed processors and memory as well as a stateof-the-art numerical technique known as adaptive mesh refinement which allows focus of computations in critical areas of the fbw [6], [7], [8], [9].

Another possible device configuration for DNA extraction is a pillar chip which is a shallow microchannel with a dense array of pillars in the fbw. This configuration lends itself to a more tractable $2 \mathrm{D}$ problem. In the right image of Fig. 1 we show the pressure in a 2D continuum model of fbw in a pillar chip. A magnified image would detail deffection of lines of constant pressure due to the pillar array.

An even simpler 2D problem with a small array of pillars can be considered to obtain relevant information for the full 3D problem. Such a model can be used as a testbed for developing the relevant physics and chemistry in the broader scale problem. We have been able to develop the physics in the algorithm for a simple two-dimensional packed array channel and capture the fundamental dynamics of a DNA molecule fbwing in a closely packed geometry (see Fig. 2). In this result the polymer enters the channel from the left and wraps around the first post it encounters. It is slowly worked off the surface by both Brownian motion and the pull of viscous forces at the loose end. It completely breaks free and proceeds out the channel with the fluid, interacting with surfaces along the way.

\section{CONCLUSION}

We have demonstrated a simulation capability for DNA molecular fbw in a microchannel configuration used for extraction. From here we can build up the model with additional physics and chemistry along with the numerical resolution enhancements. Specifically, we will incorporate a method for "sticking" using shortrange forces (van der Waals) to approximate chemical and electrostatic interactions. We will also use adaptive mesh refinement for additional scalability.

\section{ACKNOWLEDGMENT}

Work at the University of California, Davis was partially supported by the US DOE MICS Division under contract number DE-FG02-03ER25579.

\section{REFERENCES}

[1] D. Trebotich, P. Colella, and G.H. Miller, "A stable and convergent scheme for viscoelastic fbw in contraction channels," J. Comp. Phys., 2005, to appear.

[2] D. Trebotich, G.H. Miller, P. Colella, D. T. Graves, D. F. Martin, and P. O. Schwartz, "A tightly coupled particle-fluid model for DNA-laden fbws in complex microscale geometries," in M.I.T. Conference Proceedings, K. J. Bathe, Ed., Oxford, 2005, Elsevier, to appear.

[3] H. Johansen and P. Colella, "A Cartesian grid embedded boundary method for Poisson's equation on irregular domains," J. Comp. Phys., vol. 147, no. 2, pp. 60-85, December 1998.

[4] P. McCorquodale, P. Colella, and H. Johansen, "A cartesian grid embedded boundary method for the heat equation on irregular domains," J. Comp. Phys., vol. 173, pp. 620-635, 2001.

[5] J. B. Bell, P. Colella, and H. M. Glaz, "A second-order projection method for the incompressible Navier-Stokes equations," $J$. Comp. Phys., vol. 85, pp. 257-283, 1989.

[6] M. Berger and J. Oliger, "Adaptive mesh refi nement for hyperbolic partial differential equations," J. Comput. Phys., vol. 53, pp. 484-512, Mar. 1984.

[7] M. J. Berger and P. Colella, "Local adaptive mesh refi nement for shock hydrodynamics," J. Comput. Phys., vol. 82, no. 1, pp. 64-84, May 1989.

[8] J. B. Bell, M. J. Berger, J. S. Saltzman, and M. Welcome, "A three-dimensional adaptive mesh refi nement for hyperbolic conservation laws," SIAM Journal on Scientific Computing, vol. 15, pp. 127-138, 1994.

[9] A. S. Almgren, J. B. Bell, P. Colella, L. H. Howell, and M. J. Welcome, "A conservative adaptive projection method for the variable density incompressible Navier-Stokes equations," $J$. Comput. Phys., vol. 142, no. 1, pp. 1-46, May 1998. 

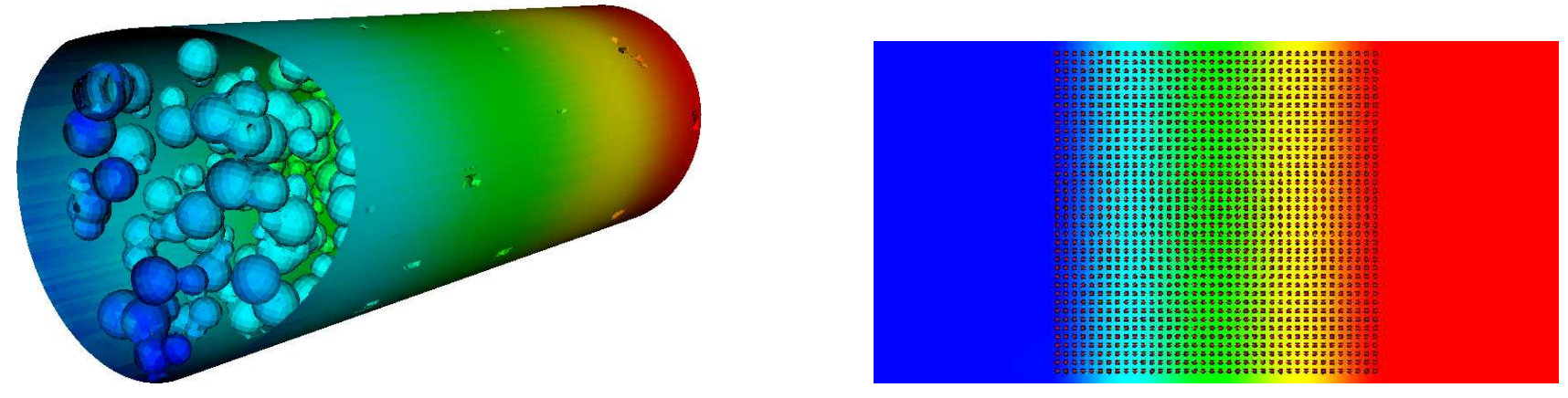

Fig. 1. Pressure data. (L) 3D continuum model for packed bed reactor geometry (cutaway). (R) 2D continuum model for pillar chip.
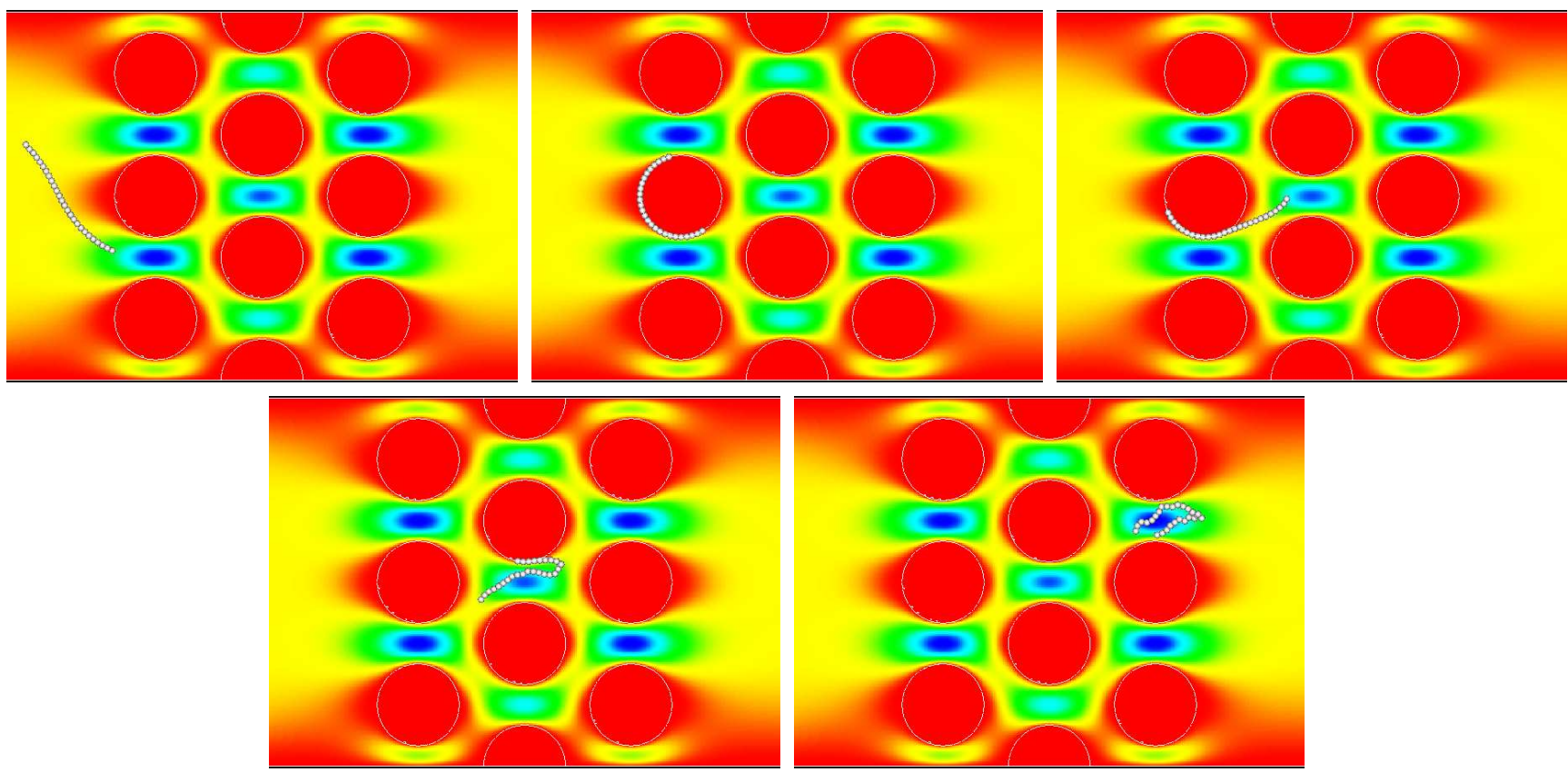

Fig. 2. Time sequence of genomic DNA fbwing in 2D model of packed bed reactor PCR chamber. DNA molecule enters from left in frame 1, then wraps around bead in frame 2, is loosened by Brownian and hydrodynamic forces in frame 3 and is swept out of the chamber by the fbw fi eld in frames 4 and 5. Color map indicates underlying velocity fbw fi eld - fast (blue), slow or reversed (red). 